SKIN CANCER

\title{
Setting the stage for cutaneous SCC
}

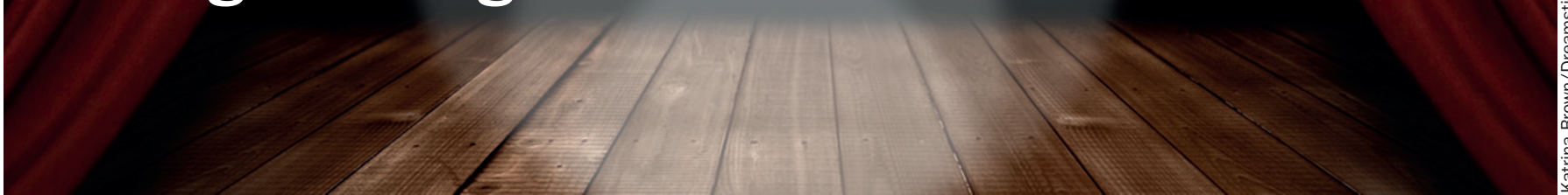

$\mathrm{n}$ the USA, over 3 million cases

of nonmelanoma skin cancer are

diagnosed each year, with over 700,000 cases being cutaneous squamous cell carcinoma (CSCC). Although most patients with CSCC have an excellent prognosis after surgical intervention, it has been known for decades that a small, but significant subset of patients with CSCC are at an increased risk of local recurrence, metastases, and ultimately die from the disease. Several clinical and histological risk factors are associated with increased recurrence, including perineural or lymphovascular invasion, poorly differentiated tumours, a diameter $>2 \mathrm{~cm}$ beyond the dermis, and location of the tumour on the ear or lip. Despite this knowledge, a consistent definition of highrisk disease and its associated prognosis is lacking. Moreover, not much evidence exists to guide nodal staging and adjuvant therapy for patients at highest risk.

To date, the American Joint Committee on Cancer (AJCC) and the International Union Against Cancer (UICC) have both published revised tumour staging systems in 2010, based on expert consensus of data derived from mostly small case series. The majority of poor outcomes occurred in patients with tumours classified as T2 according to the AJCC staging system. An alternative staging system-Brigham and Women's Hospital (BWH) - was developed to subdivide better the T2 tumours and differentiate low risk from high-risk patients within this group; initial results showed this alternative system offered improved homogeneity and monotonicity over the AJCC system.

Chrysalyne Schmults and colleagues compared the AJCC, UICC and BWH staging systems in terms of distinctiveness, homogeneity and monotonicity. They also wanted to validate the BWH staging system in a larger patient cohort. Schmults highlights the pre-existing challenges. "We had no criteria for defining high risk and no prognostic estimates for different combinations of risk factors. We did notand still do not-know which patients with CSCC should have nodal staging or adjuvant therapy after surgery." In light of this situation, the researchers wanted to better define which patients with CSCC had high-risk disease that would warrant staging or treatment beyond surgery.

In a previous study, Schmults and coauthors had generated a preliminary version of the staging system now termed the BWH staging system. They found that four risk factors were the most important contributors towards poor outcomes: diameter $>2 \mathrm{~cm}$, depth of tumour invasion beyond subcutaneous fat, poor histological differentiation, and perineural invasion. Tumours with none of these risk factors were designated $\mathrm{T} 1$, those with one risk factor were designated $\mathrm{T} 2 \mathrm{a}$, those with 2-3 risk factors designated $\mathrm{T} 2 \mathrm{~b}$, and those with all four risk factors or bone invasion were classified as T3. By conducting a 10-year retrospective single-institution cohort analysis of patients with CSCC, the research team assessed whether the previous staging system they had developed could be improved, and whether it had advantages over the current AJCC and UICC staging systems. All tumours in the cohort were staged according to BWH, AJCC, and UICC staging systems. Cox proportional hazards and Fine and Gray competing risk modelling were used to determine which patient factors were indepdendently associated with various outcomes.

Schmults highlights the key findings of the trial: "A large majority of tumours were categorized into low $\mathrm{T}$ stages (T1 and T2) in the AJCC and UICC staging systems and many poor outcomes also occurred in these low stages-crucially this indicates these systems do not adequately cluster high-risk tumours into high $\mathrm{T}$ stages. Conversely, in the BWH staging system, poor outcomes are better clustered within the highest stages (T2b and T3). Only 5\% of tumours were T2b or T3, but this small subset contained $70 \%$ of nodal metastases and $83 \%$ of deaths due to CSCC."

Importantly, this study is the "first to apply outcome data from a large cohort to AJCC and UICC staging systems to test their validity. This testing shows deficiencies in AJCC and UICC staging due to overly restrictive criteria for high-stage disease resulting in very few tumours in the upper stages and most poor outcomes subsequently occurring in low-stage tumours." Schmults continues, "the BWH staging system remedies this to a large degree by clustering the majority of poor outcomes in high tumour stages. Our study also provides the first prognostic estimates by tumour stage for SCC."

Schmults summarizes future plans for ongoing work in this area. "Further validation of the staging system is underway in a population-based cohort and other hospital-based cohorts. We are also beginning to search for genetic factors that are associated with poor outcomes and which may be incorporated into future prognostic models or staging systems."

\section{Lisa Hutchinson}

\footnotetext{
Original article Karia, P. S. et al. Evaluation of American Joint Committee on Cancer, International Union Against Cancer, and Brigham and Women's Hospital tumor staging for cutaneous squamous cell carcinoma. J. Clin. Oncol. doi:10.1200/JC0.2012.48.5326
} 